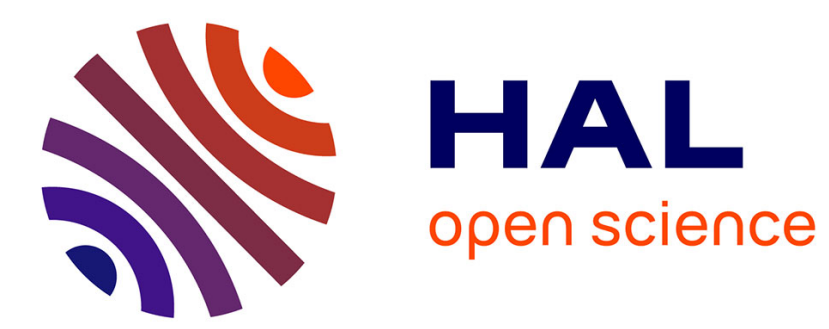

\title{
Au prix du sens, voire des valeurs
}

Christophe Dansac

\section{To cite this version:}

Christophe Dansac. Au prix du sens, voire des valeurs : Ce que peut faire le management à la ressource humaine des associations. Empan, 2017, 107 (3), 10.3917/empa.107.0058 . hal-01632442

\section{HAL Id: hal-01632442 \\ https://hal.science/hal-01632442}

Submitted on 10 Nov 2017

HAL is a multi-disciplinary open access archive for the deposit and dissemination of scientific research documents, whether they are published or not. The documents may come from teaching and research institutions in France or abroad, or from public or private research centers.
L'archive ouverte pluridisciplinaire HAL, est destinée au dépôt et à la diffusion de documents scientifiques de niveau recherche, publiés ou non, émanant des établissements d'enseignement et de recherche français ou étrangers, des laboratoires publics ou privés. 
Au prix du sens, voire des valeurs -

Ce que peut faire le management à la ressource humaine des associations

\section{Une approche volontairement négative face à des idéologies trop optimistes}

Sans prétendre à l'exhaustivité, cette contribution, polémique au sens positif du terme, s'appuiera sur des travaux issus de la littérature internationale ainsi que sur des études françaises pour mettre en évidence quelques effets négatifs que peut avoir le management sur le fonctionnement des associations et sur les personnes qui y contribuent. L'auteur prie donc à l'avance ses lecteurs de bien vouloir excuser sa posture, qui fait le choix prospectif inhabituel de ne considérer que quelques signaux faibles, négatifs, et de pointer les menaces qu'ils font peser sur l'idéal associatif. Pour planter le décor de cette réflexion, nous donnerons d'abord une définition du managérialisme, pour ensuite explorer comment le management influence trois catégories de ressources humaines du monde associatif, les salariés, les bénévoles, et les volontaires.

Klikauer (2015) synthétise les travaux critiques sur le management en proposant une vision particulière du managérialisme. Pour lui, il s'agit de l'idéologie qui prête au management la capacité à traiter tous les problèmes de la société de la même façon qu'ont été prétendument résolus les problèmes de production au cours du développement industriel. Dès les travaux de Taylor sur l'organisation du travail, les partisans du management ont su donner à la discipline une coloration scientifique et positiviste qui a ensuite été favorable à son expansion. Dans leur approche, l'évaluation - composante essentielle du contrôle - est centrale, et la mesure - qui plus est objective - est fantasmée comme étant toujours possible. Ainsi, les managers pourraient optimiser, avec les mêmes méthodes, le fonctionnement de l'industrie comme celui de l'éducation, des services sociaux, ou encore celui de l'organisation générale de la société, et donc des politiques publiques.

Cette idéologie parvient progressivement à s'imposer dans le monde associatif, certains auteurs ayant montré à quel point certaines organisations approchent dans leur fonctionnement celui des entreprises capitalistes classiques (Hély, 2009). Ce rapprochement résulte selon DiMaggio et Powell (1983) de l'isomorphisme 
institutionnel qui comprend trois mécanismes : 1) l'isomorphisme normatif résulte de la place croissante des compétences administratives et comptables et de la formation en management des professionnels qui les assument ;2) l'isomorphisme mimétique, où les modèles des organisations emportant les financements sont copiés pour les concurrencer; et 3) l'isomorphisme coercitif vient de la pression politique (formelle ou non) qui s'exerce sur les associations de par la façon dont l'agenda néolibéral favorise le managérialisme. Ainsi le managérialisme qui empreint les politiques publiques contribue à implanter le managérialisme dans les secteurs qui dépendent d'elles. Ce rapprochement est pourtant souvent dénié dans le milieu associatif, qui continue à mettre en avant sa «capacité à prendre en compte des valeurs autres que financières » et un idéal associatif particulièrement fondé sur la dimension démocratique (Hély \& Moulévrier, 2009) et les valeurs humanistes portés par l'associationnisme qui permettrait d'éviter conflits et tensions. Et ceci alors même que la démocratie associative est souvent mise à mal (Dansac, Gontier, et al., 2013) et que les associations sont loin d'être exemptes de conflits liés à leurs modes de gestion (Peyre, 2005).

Dans une étude portant sur 200 associations (aux USA), Hwang et Powell (2009) ont montré que le niveau de rationalisation organisationnelle variait selon plusieurs facteurs, et qu'au nombre de ceux-ci, on comptait non seulement le niveau de professionnalisation de la main d'œuvre, mais aussi la présence d'un management. Ils montrent également que la rationalisation est plus forte pour les structures dont les managers ont bénéficié de formations en management (de type MBA) que pour celles où ils proviennent du champ de spécialité de l'association. À mesure qu'elles se professionnalisent, les associations évoluent du plaidoyer vers l'instrumental, dans un mouvement qui s'accompagne d'une standardisation, d'une diminution de l'expérimentation, et d'un accroissement du recours extérieur à des structures d'audits. La taille, enfin, est un déterminant de la mise en place de la professionnalisation et de la rationalisation organisationnelle qui l'accompagne.

\section{Du managérialisme à l'inflation mécanique de la taille des organisations}

L'augmentation de la taille des organisations peut être vue comme étant le facteur initial du besoin de management, et comme portant intrinsèquement les germes de la déviation 
du projet associatif initial (Dansac, Vachée, \& Gontier, 2016; Sundblom, Smith, Selle, Dansac, \& Jensen, 2016). Or, d'une part cette augmentation de la taille correspond à une pression des services de l'État (un directeur nous rapportait que lors d'une réunion des associations du secteur médico-social avec une ARS, le fonctionnaire avait posé comme préambule de la réunion que les interlocuteurs autour de la table étaient trop nombreux). D'autre part, elle rejoint la préoccupation des élus locaux quant à la fiabilité et la robustesse des structures susceptibles de porter les missions qui leur ont été dévolues lors de la décentralisation (dans l'attribution de la gestion d'une structure d'hébergement temporaire pour adulte nouvellement créée dans une sous-préfecture, le choix d'une grosse association allant au-delà de la région administrative plutôt que d'une association locale plus petite a été justifié par cette apparente solidité). L'adage selon lequel on ne prête qu'aux riches trouve aussi sa réalisation dans les rapports entre les associations et leurs financeurs. La mise en concurrence des associations par le new public management, favorise ainsi les fusions, les absorptions et les restructurations, pour aboutir à des organisations plus importantes, mieux à même de missionner des spécialistes de la conquête des marchés publics.

L'accroissement des structures en taille et en complexité contribue à l'éloignement de la décision managériale des travailleurs au contact des publics. Un éducateur spécialisé, suite à la restructuration des établissements médico-sociaux sur un département rural éloigné de la métropole régionale, rapportait ainsi en entretien : «le directeur, il passe beaucoup de temps en réunion, loin de [Établissement], il est presque en permanence à [Métropole régionale], heureusement que notre chef de service essaye de défendre les manières de travailler qu'on a ici, parce que sinon, on nous imposerait plein de choses qu'on ne veut pas dans l'équipe ». C'est ainsi parfois le management de proximité qui protège des décisions managériales aveugles qui viendraient des organes gestionnaires centralisés, dont le rôle est justement la rationalisation organisationnelle. Cette protection par le management de proximité peut toutefois être mise à mal du fait que le managérialisme entraine le recrutement de managers qui ne sont pas issus du métier, l'idéologie postulant que leurs compétences génériques peuvent être appliqués dans n'importe quel secteur: Un président d'association d'éducation populaire, historiquement militant du secteur, témoignait avoir privilégié au recrutement d'un 
directeur un gestionnaire sans connaissances du milieu en raison de la complexification des tâches administratives.

Dans certains cas, la mécanique de la rationalisation fait fi du management de proximité, comme le montre ce témoignage d'un ancien cadre d'un établissement médico-social ayant démissionné justement parce qu'il ne s'y retrouvait plus au niveau des valeurs. Il était employé dans une association régionale implantée sur de nombreux sites dont certains distants de la métropole, par des établissements de natures diverses. Le siège de l'association choisit de consolider l'activité d'un ESAT qu'elle dirigeait, et d'optimiser le fonctionnement d'ensemble des structures en leur faisant profiter de ses services : «à [nom de la métropole régionale] il a été décidé qu'on aurait les repas livrés, et qu'on aurait juste à les faire réchauffer, ça réglait un problème, celui de la cuisine qui n'était pas tout à fait aux normes, mais par contre, on ne pouvait plus faire la cuisine avec les résidents, et du coup, il y a toute une part du sens de ce que c'est que manger ensemble, en ayant préparé le repas, qui n'était plus là. Nous, les éduc, on n'a pas été consultés, et ils n'ont pas vu l'aspect éducatif, ce que ça donnait au niveau du collectif et de son fonctionnement $\gg$. Dans ce cas précis, il y a eu perte de sens du travail pour le salarié, mais on peut considérer qu'indirectement, ce sont aussi les usagers qui pâtissent du management.

\section{De plus grosses associations ne font pas forcément des salariés plus heureux...}

Dans la dernière décennie, les fusions se sont multipliées en France, et le monde associatif a produit nombre de guides sur les bonnes manières d'y procéder notamment sur le plan des ressources humaines. La sensibilité à la question du bien être des salariés de l'ESS a d'ailleurs mené la mutuelle CHORUM à créer un «centre de ressources et d'action pour le développement de l'emploi de qualité », le CIDES. Et à mettre en place en 2013 un baromètre de la qualité de vie au travail $(\mathrm{QVT})^{1}$ dont les résultats instructifs ont notamment conduit le Mouvement Associatif à lancer en 2014 un prix pour l'emploi de qualité ${ }^{2}$.

\footnotetext{
${ }^{1}$ http://www.chorum-cides.fr/ressource/barometre-sur-la-qualite-de-vie-au-travail-dans-less-les-resultats/ ${ }^{2} \mathrm{http} / / / \mathrm{www}$.prix-emploi-qualite.org/
} 
Car un des effets du managérialisme selon Klikauer, c'est de consolider la position des managers, et de précariser les travailleurs spécialistes. Pour sécuriser le fonctionnement des organisations, les premiers utilisent leurs compétences génériques pour formaliser et standardiser l'intervention des seconds, réduisant leur autonomie et les rendant interchangeables. Les grandes organisations offrent des perspectives de carrières intéressantes pour ceux qui sont dans des fonctions support, mais n'offrent pas pour autant reconnaissance et sécurité à ceux qui sont pourtant indispensables à l'exercice de leurs missions, le cœur du métier étant précarisé. Illustration de ce renversement des valeurs, l'analyse des résultats du baromètre QVT 2013 montre que la confiance dans l'avenir professionnel au sein de la structure est plus élevée chez les salariés ayant des fonctions de support (comptabilité, logistique, informatique, marketing, communication) que pour ceux qui sont dans des fonctions d'accompagnement du public (63\% contre 57\%). On retrouve d'ailleurs la même différence en ce qui concerne le sentiment d'avoir du soutien de la part de son encadrement, les salariés de l'accompagnement étant plus nombreux (30\%) que ceux des fonctions support (24\%) à répondre qu'ils ne bénéficient pas de soutien.

Dans ce même baromètre 2013,30\% des salariés signalaient que leur organisation avait subi récemment une fusion ou un regroupement. Ces salariés ont une estimation de leur qualité de vie au travail plus faible que ceux qui n'en ont pas vécu. Là encore, parmi ceux ayant vécu une fusion, les salariés ayant des fonctions support sont proportionnellement plus nombreux à considérer qu'elle a eu un impact positif sur leur travail ou sur la pérennité de leur emploi que les salariés en charge de l'accompagnement des publics. Autrement dit, la complexification des organisations profite peu à ceux qui sont au cœur de leurs missions, alors qu'elle en renforce la technostructure.

Notons enfin que dans la seconde version de ce baromètre, réalisée en 2017, pour près de la moitié (46\%), les salariés considèrent que la qualité de vie au travail dans leur poste s'est dégradée dans les dernières années, et que la dégradation est vécue comme plus forte dans les organisations de plus de 500 salariés. 
Mais l'augmentation de la taille, que ce soit par accroissement de l'activité ou par des fusions, et la rationalisation organisationnelle n'affectent pas seulement les salariés, elle modifie en profondeur le rapport au bénévolat.

\section{$\underline{\text { Associations managées ne riment pas forcément avec bénévoles impliqués }}$}

Accroissement de la taille et complexification des associations entrainent bien souvent des difficultés à trouver des dirigeants bénévoles capables et désireux d'assumer des responsabilités juridiques de plus en plus lourdes. À l'image des associations américaines «sans membres » qui mettent la démocratie hors-jeu (Skocpol, 2003), certaines associations médicosociales sont déjà dépourvues de bénévoles autres que les dirigeants (voire d'adhérents), et dans les autres secteurs, la distance entre bénévoles dirigeants et bénévoles de terrain s'accroit à mesure que la technicité des salariés vient remplacer la décision collective (Kreutzer, 2009; Dansac, Gontier, et al., 2013). Distance qui a son tour rend plus difficile la succession des dirigeants. Heureusement, pourrait-on dire ironiquement, le management peut résoudre ce problème: la «filialisation» dans le cadre du groupe $\operatorname{SOS}^{3}$ va dans ce sens. Elle propose de remplacer l'assemblée générale de personnes physiques par des personnes morales (les associations du groupe), ce qui permet de remplacer un conseil d'administration en mal de renouvellement de bénévoles par un seul de ses membres, éventuellement le président. La filialisation boucle ainsi le processus, permettant à la nouvelle filiale allégée en participation bénévole - de bénéficier de la puissance managériale des services de support du groupe, tout en conservant les avantages du statut associatif.

Une des forces du monde associatif, régulièrement mise en avant, c'est le travail fourni par ses bénévoles. Il y a de grandes disparités en ce qui concerne l'attractivité des associations, mais qu'elles en disposent ou non, les associations semblent toujours en demande de main d'œuvre supplémentaire. Et ceci est particulièrement le cas en ce qui concerne l'implication des jeunes, qui généralement s'investissent assez peu dans les organisations historiques ou de grande taille, où ils ont du mal à trouver leur place (Dansac, Bordes, Gontier, \& Vachée, 2013). Pour attirer ceux-ci, certains font recours à

\footnotetext{
${ }^{3} \mathrm{Cf}$. http://www.associations.groupe-sos.org/rejoignez-groupe-sos
} 
des programmes (importés des USA) dont on peut questionner la compatibilité avec leurs valeurs. On a ainsi vu des jeunes de Paris, Marseille ou Bordeaux fournir « 4 heures de [leur] temps en musique et en équipe » pour repeindre des équipements sociaux ou mettre en place des jardins solidaires au profit d'associations à vocation sociale ou socioculturelle, et ce en échange d'un concert privé gratuit de stars internationales ${ }^{4}$.

Le renoncement au caractère désintéressé du don de soi qui caractérise le monde associatif est encore plus clairement acté dans le cadre du dispositif de volontariat en service civique, où pour «satisfaire le besoin d'engagement des jeunes», une indemnisation (moins de 600 euros) leur est proposée en échange de la réalisation d'une mission d'intérêt général. Celui-ci a tout de même comme conséquence de faire travailler des jeunes, y compris dans des associations qui combattent la pauvreté et ses conséquences - parfois bien au-delà des 25 heures hebdomadaires minimales - contre une indemnité qui les met sous le seuil de pauvreté (Bacou, Dansac, Gontier, \& Vachée, 2014).

Ces deux exemples démontrent comment l'éthique de responsabilité, au fondement du managérialisme, peut parfois prendre le dessus sur l'éthique de conviction (Weber, 1922) : les moyens adaptés à une situation (de pénurie) sont utilisés sans prise en compte d'éventuels « dommages collatéraux » qu'ils pourraient entraîner.

\section{L'évolution des modes de financement, menace ou opportunité ?}

Les changements de modes de financement par l'État de l'action associative ont fortement bouleversé les organisations. À l'heure où commencent à se développer, toujours sur le modèle anglo-saxon, le financement par les obligations à impact social (SIBs), on peut questionner l'influence qu'auront ces contrats sur l'intervention sociale dont ils sont censés régler les problèmes de financement grâce à des investissements privés. Le Haut Conseil à la Vie Associative dans son avis du 2 mars 2016, considère que les «SIBs peuvent être une opportunité pour former une culture de contrôle et

\footnotetext{
${ }^{4}$ Cf. http://www.rockcorps.com/ ou https://www.facebook.com/orangerockcorps.communitymanager?fref=ts
} 
d'évaluation en matière de prestation de services sociaux. Une évaluation indépendante et robuste peut être bénéfique pour toutes les parties prenantes en tant qu'elle permet d'identifier ce qui marche bien dans les SIBs et ce qui fonctionne moins bien, ainsi que les conséquences inattendues, positives ou négatives. » (p.3) En cela, ils rejoignent les conclusions de nombreux chercheurs qui soulignent le caractère positif d'une évaluation sur le résultat plutôt que le processus (cf. Fraser et al., 2016), mais oublie le surcroît d'évaluation généré soutenant le managérialisme. Ils omettent aussi un risque pointé par les chercheurs, celui de permettre que le bénévolat qui entre généralement en jeu dans l'intervention sociale soit mis au service du profit financier. Rien ne permet pour l'instant de prédire comment les bénévoles verront leur investissement gratuit servir le rendement financier au profit des investisseurs.

Le paradigme de l'ESS, qui promeut entreprenariat social, coopératives et fondations, permettra peut-être de faire évoluer les associations et d'y clarifier les le rôle et le statut des bénévoles. Celles-ci y gagneront peut-être une plus grande simplicité en évitant ce que Kreutzer et Jager (2010) décrivent comme une source des conflits internes : les identités organisationnelles multiples qui résultent de la cohabitation de bénévoles et de salariés, de managers généralistes et d'intervenants sociaux spécialisés. L'inflation de la taille des associations et la dégradation corollaire du sens du travail sous l'effet du managérialisme conduiront peut-être les salariés associatifs à réinvestir les syndicats, dont Baines, Charlesworth, et Cunningham (2013) montrent le rôle protecteur vis-à-vis du new public management.

Dans le projet Gouvernance des Associations et Rapports de Pouvoirs (Dansac, Gontier, et al., 2013), nous avions repéré que face aux pressions extérieures, ce sont les valeurs portées par l'association, et surtout par ceux qui la défendent en les incarnant avec charisme auprès des extérieurs partenaires ou tutelles, qui pouvaient permettre la résistance. Réflexion sur les valeurs et formation, tant pour les bénévoles que les salariés opérationnels et le management, sont sans doute les clés pour permettre que l'ensemble des ressources humaines associatives s'allie, comme Baines et al. le suggèrent, pour tirer parti du new public management en détournant la mesure, afin de lui donner du sens en ce qui concerne les valeurs de l'intervention sociale. 


\section{Références}

Bacou, M., Dansac, C., Gontier, P., \& Vachée, C. (2014). Le volontariat dans l'animation: vers une déprofessionnalisation du secteur au nom de l'engagement. Agora Débats/Jeunesses, 67(2), 37-51.

Baines, D., Charlesworth, S., \& Cunningham, I. (2013). Fragmented outcomes: International comparisons of gender, managerialism and union strategies in the nonprofit sector. Journal of Industrial Relations, 56(1), 24-42.

Dansac, C., Bordes, V., Gontier, P., \& Vachée, C. (2013). Renouvellement et Rajeunissement des Instances Bénévoles Dirigeantes. Figeac: LRPMip, IUT de Figeac / UMR EFTs.

Dansac, C., Gontier, P., Vachée, C., Maisonneuve, C., Goutas, N., \& Taillandier, A. (2013). Gouvernance des Associations et Motivations des Bénévoles. Figeac: LRPMip, IUT de Figeac / Région Midi-Pyrénées / CPCA Midi-Pyrénées.

Dansac, C., Vachée, C., \& Gontier, P. (2016). Du bénévole militant à l'usager consommateur: Transformation des modes d'engagement sous l'effet du développement d'une association. In F. Lebon \& E. De Lescure (Éd.), L'éducation populaire au tournant du XXIè siècle (p. 247-262). Créteil, France: Croquant (Edition du).

DiMaggio, P. J., \& Powell, W. W. (1983). The Iron Cage Revisited: Institutional Isomorphism and Collective Rationality in Organizational Fields. American Sociological Review, 48(2), 147-160.

Fraser, A., Tan, S., Lagarde, M., \& Mays, N. (2016). Narratives of Promise, Narratives of Caution: A Review of the Literature on Social Impact Bonds. Social Policy \& Administration. 
Hély, M. (2009). Les métamorphoses du monde associatif. Paris: Presses universitaires de France.

Hély, M., \& Moulévrier, P. (2009). «Économie sociale et solidaire : quand les sciences sociales enchantent le travail. Idées économiques et sociales, 158(4), 30.

Hwang, H., \& Powell, W. W. (2009). The Rationalization of Charity: The Influences of Professionalism in the Nonprofit Sector. Administrative Science Quarterly, 54(2), 268-298.

Klikauer, T. (2015). What Is Managerialism? Critical Sociology, 41(7-8), 1103-1119.

Kreutzer, K. (2009). Nonprofit governance during organizational transition in voluntary associations. Nonprofit Management and Leadership, 20(1), 117-133.

Kreutzer, K., \& Jager, U. (2010). Volunteering Versus Managerialism: Conflict Over Organizational Identity in Voluntary Associations. Nonprofit and Voluntary Sector Quarterly, 40, 634-661.

Peyre, M. (Éd.). (2005). Le livre noir de l'animation socioculturelle. Paris: L'Harmattan.

Skocpol, T. (2003). Diminished democracy: from membership to management in American civic life. Norman: University of Oklahoma Press.

Sundblom, D., Smith, D. H., Selle, P., Dansac, C., \& Jensen, C. (2016). Life cycles of individual associations (Chap. 38). In D. H. Smith, R. A. Stebbins, \& J. Grotz (Éd.), The Palgrave handbook of volunteering, civic participation, and nonprofit associations. New York, NY: Palgrave Macmillan.

Weber, M. (1922). Economie et société (édition en 2 tomes, 1995). Paris: Pocket. 


\section{$\underline{\text { Auteur }}$}

Christophe DANSAC

Directeur du Laboratoire de Recherche Pluridisciplinaire du Nord-Est Midi-Pyrénées (LRPMip), groupe Organisations Non Orientées vers le Profit et Gouvernance.

Enseignant-chercheur à l'IUT de Figeac, Université Toulouse 2 Jean Jaurès, 2 avenue de Nayrac, 46100 FIGEAC (christophe.dansac@ univ-tlse2.fr).

\section{Résumé}

Cette contribution se base sur une définition du managérialisme comme étant l'idéologie qui prête au management la capacité à traiter tous les problèmes avec les mêmes méthodes, en plaçant au cœur des solutions l'évaluation. Elle explore certaines de ses conséquences dans les politiques publiques comme au sein des associations, en reliant la taille de celles-ci à leur rationalisation organisationnelle. Elle pointe certains effets de ces facteurs sur le rapport à l'organisation de trois catégories de ressources humaines du monde associatif, les salariés, les bénévoles, et les volontaires.

\section{$\underline{\text { Mots-clefs }}$}

Managérialisme, travailleurs associatifs, intervention sociale, bénévolat, politiques publiques. 